als irgend Jemand. Die Regierung hat die Möglichkeit einer genauen Analyse jedenfalls sorgfältigst geprüft, und wenn davon die Zurïckweisung von Lieferungen im Werte von etwa $50000 \mathrm{M}$. abhängt, so sehen Lieferant und Regierung auf genaue Untersuchung, die nicht jungen Herren überlassen wird. Man kann also ruhig auf die erste Dezimalstelle solcher Resultate schwören.

Ich habe Lunge in Bezug auf seine hochnitrierte Schie日wolle keinen Irrtum vorgeworfen, im Gegenteil ich betonte ndie Tatsachen anzuzweifeln, wāre lächerlich". GewiB, keiner der Kopfschüttler hat je Lungesche Schießwolle von 13,9 Proz, in Händen gehabt, die nach einigen Tagen so merkwürdig zurückgeht; sie sind zwar Chemiker hervorragender Fabriken, waren aber wohl nicht geschickt genug, um sie nachmachen zu können, deshalb $z$ weifelten sie, daß Lunges Produkt reine Schiebwolle sei.

Daß ich mir gestattete zu erwähnen, ich hätte "Ursache zu glauben" ${ }^{2}$ ), es sei seitdem versuchsweise stabile Schießwolle von 13,9 Proz. gemacht worden, ist mein gutes Recht, auch wenn Lunge dem keine Beachtung schenken will. Er sagt selbst, dab "die Fabriken der Sprengstoffindustrie durchweg eine öffentliche Erwähnung ihrer Erfahrungen nicht gestatten ", und ich kann wohl hinzusetzen, weder mit Lunge in einen Federkrieg geraten, noch Konkurrenten die Mittel zur Nachprüfung an die Hand geben wollen.

$\mathrm{Da}$ ich verschiedentlich anderer Meinung bin, als Lunge und Bebie, ja sogar als Vieille, ist nicht so sakrilegisch, als Lunge es darstellt. Vieilles Maximum wird von Niemandem als solches anerkannt; er hat unter Bedingungen gearbeitet, bei welchen ein höherer Stickstoffgehalt nicht zu erreichen war. Ich habe auch Lunge brieflich darauf aufmerksam gemacht, daß die amtliche Specifikation der Vereinigten Staaten ${ }^{3}$ ) bei rauchlosem Pulver für die Marine verlangt: „Der Prozentgehalt an Stickstoff der unlöslichen Nitrocellulose muB 13,30土0,15 sein". Es ist jedem Fabrikanten klar, daß man da manchmal höher als 13,45 Proz. kommen muß und sich durch Vermengen mit ebenso leicht vorkommender Nitrocellulose onter 13,30 Proz. hilft. Auch in dem Corditprozesse, wo Lunge und ich als Sachverständige fungierten, hat Sir Henry Roscoe bezeugt, er habe im großen Maßstabe Schieß-

2) Ich gebrauchte lcider eine englische Redensart, aber Lunge weiß sehr wohl, sie bedeute, ich habe Nachricht aus authentischer Quelle".

3) -Wiltoughby Walke, Lectures on Explosives, 1897, S. 330. wolle mit 13,60 Proz. (13,70 Proz. aschenfrei) hergestellt. (Frage No. 1637 und 1638 des amtlichen Protokolles.) Ich befinde mich also mit meiner Häresie in guter Gesellschaft,

In Bezug auf lösliche Nitrocellulose von mehr als 12,9. Proz. glaubt Lunge weder mir, noch Schüpphaus, dem er sogar sagt, „das Papier ist ja geduldig". Das tut mir aufrichtig leid, denn ich bätte gedacht, daB Lunge und ich, die einander seit 18 Jahren nicht nur schätzen, sondern wie ich hoffe, auch achten gelernt haben, nicht jedesmal eine Beglaubigung von zwei Zeugen vorweisen müssen, wenn wir etwas behaupten. Das würde eine böse Zeit für die Wissenschaft werden, wenn das. Wort von Leuten nichts mehr gilt, die einen guten Ruf zu verlieren haben. Ein wissenschaftlicher $\mathrm{Be}-$ weis ist deren Behauptung nicht, aber sie verdient Achtung selbst in einer wissenschaftlichen Kontroverse.

London, 7. März 1903.

\section{Über die Herstellung der Zinkretorten und deren Verhalten im Feuer.}

(Dritte Mitteiluag über den Ton von St. Louis.) Von Dr. Otto Mühlhaouser.

Fine der hauptsächlichsten Anwendungen, welche der Ton von St. Louis erfährt, ist diejenige zur Herstellung von Zinkretorten. Es sind dies bekanntlich röhrenförmige $\mathrm{Ge}$ fäße, in welchen man die Reduktion des Metalloxyds mit Kohle vornimmt und gleichzeitig das Zink abdestilliert. Da dieser kombinierte Prozeß bei hoher Hitze ausgeführt wird und die GefäBe im Zinkofen den verschiedenartigsten pyrochemischen, mechanischen . und physikalischen Einfüssen bez. Angriffen ausgesetzt sind, so stellt man an die Zinkretorten die allergröBten Anforderungen in Bezug auf allgemeine Widerstandsfähigkeit und Haltbarkeit.

Von einer Zinkretorte verlangt man im allgemeinen: einen ziemlich bedeutenden Grad von Schwerschmel zbarkeit (über Segerkegel 30), Widerstandsfähigkeit gegen Berührungsmittel (Flugstaub, Schlacke im Innern der Retorte), Zähigkeit und mechanische Festigkeit resp. Widerstandsfähigkeit gegen mechanische Einwirkungen (StoB und Schlag), Unempfindlichkeit gegen Temperaturschwankungen bez. Fähigkeit, plötzliche Abkühlung, wie sie beim Chargieren der Retorten eintritt, zu ertragen, hohen Grad vờ Undurchlāssigkeit für Zinkdämpfe, Widerstand gegen Deformation (Einbiegen im Ofen), nicht allzu starkes Nachschwinden im Feuer, lange Lebensdauer. 
Inwieweit nun die aus Ton hergestellten Gefäße den Anforderungen, die der Betrieb an sie stellt, genügen, hängt außerordentlich viel von der Zubereitung der Massen ab, deren Zusammensetzung und Aufbau aus Ton und Magermittel bez. den einzelnen Chamottekornklassen nicht willkürlich, sondern in zielbewußter Weise zu geschehen hat. Es ist der Zustand des gewetterten Bindetons, die Porosität der Chamotte, das Verhältnis, in welchem Ton und Magermittel gemischt sind, der Wassergehalt der Masse beim Pressen, die Art und Weise der Formgebung, des Trocknens, Verglühens und Einsetzens der Retorte in den Zinkofen von grö̈tem Einflub auf die Qualität der Retorte bez. deren Verhalten im Zinkofen, sodaß die einzelnen Momente, welche die Güte einer Retorte beeinflussen, in vollstem $\mathrm{MaBe}$ berücksichtigt werden müssen.

Ohne mich indessen an dieser Stelle weiter über die diesen Gegenstand berührenden Fragen verbreiten zu wollen, beschreibe ich im folgenden kurz die Herstellung der Retorten nach einem in Nord-Amerika ziemlich verbreiteten Verfahren, um daran anschliebend das Verhalten der Retorte im Feuer und deren Schicksal ausführlich zu erörtern, was, da dieser Gegenstand noch niemals eine sachgemäBe und seiner Bedeutung entsprechende Behandlung gefunden hat, von ganz allgemeinem Interesse sowohl für den Metallurgen, wie für den chemischen Technologen sein dürfte.

\section{Herstellung der Retorten.}

Herstellung der Masse. Gelegentlich der Schilderung der Herstellung von Massen aus St. Louis-Ton habe ich die Darstellung der Chamotte und auch die Zubereitung von Retortenmasse') beschriebeu. Wie erwähnt, erhält man dieselbe durch Vermahlen von $44 \mathrm{~T}$. trockenem Ton mit $56 \mathrm{~T}$. Chamotte auf ein Mehl No. 10 und Verarbeitung desselben im Tonschneider zu einem mäBig steifen Teige. Der zu Ballen verformte Teig wird in Form eines Haufens sich selbst überlassen. Nach längerem Mauken wird die Masse senkrecht abgestochen und noch einmal im Tonschneider durchgearbeitet. Die durchweg homogenen Ballen transportiert man in die Nähe der Tonpresse, wo sie aufgestapelt und nach Bedarf auf Retorten verformt werden.

Derartig zubereitete formrechte Masse hat beispielsweise folgende Zusammensetzung:

$\begin{array}{crlllll}\text { Klasse } & 8 & . & . & 0,77 & \text { Proz. } \\ - & 10 & . & . & . & 5,86 & - \\ - & 14 & . & . & . & 14,04 & - \\ - & 18 & . & . & . & 6,51 & -\end{array}$

1) Vergl. meine latzte Abhandlung Zeitschr. f. angew. Chem. 1903 S. 222.

$$
\begin{aligned}
& \text { Klasse } 20 \text {. . 27,26. Proz. } \\
& 40 \text {. . . 11,29 } \\
& 60 \text {. . } 5,32 \\
& 80 \cdot . \quad 3,56 \\
& 100 \text {. . 25,38 }
\end{aligned}
$$

Eine aus der Tonpresse austretende Probe vom Strang enthielt:

Ton und Chamotte (auf vorglühte

Substanz berechnet) . . . 78,96 Proz.

Gebundenes Wasser . . . . 5,16

Freies Wasser . . . . . . . 15,88

Herstellung der GefäBe. Zur Formgebung verwendet man eine mit Preßkopf versehene Schneckenpresse, deren Mundstück den Querschnitt der Retorte bestimmt. Die Presse befindet sich in senkrechter Stellung. Um den aus dem Mundstück austretenden, verhāltnismä日ig leicht deformierbaren 'Tonstrang zu stützen und zu führen, läßt man ihn in eine hölzerne Form eintreten, in welchem sich ein an einer Stange befestigter, mit Gewichtsausgleich versehener Teller maschinell auf- und abbewegen läßt. Will man eine Retorte herstellen, so stellt man die aus 4 Halbzylindern bestehende durch Scharniere und Keile zu einem Zylinder vereinigte Form, genau über den mit dem FuBboden in einer Ebene sich befindenden Teller, unterhalb des Mundstücks, zentrisch auf. Dann setzt man mit einem Mechanismus den durch die Stange getragenen Teller in $\mathrm{Be}$ wegung und führt denselben durch die Form hindurch bis zum Mundstück. Man setzt nun die Presse in Tätigkeit. Der Strang tritt horizontal abgeschnitten aus, stützt sich auf den Teller, welcher langsam und mit derselben Geschwindigkcit, mit welcher der Strang austritt, zurückweicht. Am Fußboden angekommen, stellt man die Maschine still, schneidet den Strang ab und entfernt Form samt Inhalt. Behufs Einsatz des Bodens schiebt man ein der GröBe desselben entsprechendes, kreisrund ausgeschnittenes Tonstück in den untersten Teil der Röhre, bringt Form samt Inhalt auf eine passende Unterlage und verschweißt den Boden mit der Röhre mittels einer Stampfe. Wollte man jetzt ohne weiteres die Form entfernen, so würde sich die Retorte deformieren. Man hat daher an der Innenseite der Form, in deren Längsrichtung, Nuten vorgesehen, in welche man entsprechend grobe Latten der Länge nach einschiebt. Dann erst öffnet man die obere Formhälfte, entfernt dieselbe und stülpt Reifen über die die Röhre stützenden Stäbe. Nach Entfernung der unteren Hälfte der Form und Freilegung der Retorten, putzt man letztere und überläßt sie nunmehr sich selbst.

Trocknen der Retorten. Nach einigen Tagen ist die Retorte so weit abgetrocknet, 
daß man auch die Stützen abnehmen kann. Die Kammern, in welchen sich die Röhren befinden, sind ventiliert, und geschieht das Trocknen in der Weise, daB man die Retorten einem Luftstrom entgegenrückt, derart, daß die trockensten zuerst, die feuchten zuletzt, mit dem einer Kalorifere entströmenden Luftstrom in Berührung kommen (Gegenstromprinzip). Ein Ventilator entzieht dem Trockenraume die feuchte Luft und führt dieselbe ins Freie. In den Trockenkammern herrscht eine Temperatur ron ca. $35^{\circ} \mathrm{C}$. Nach ca. 60-70 Tagen werden die Retorten in Gebrauch gegeben.

Eine 63 Tage der Trocknung ausgesetzt gewesene Retorte zeigte beispielsweise noch folgenden Feuchtigkeitsgehalt:

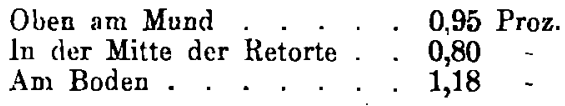

Die Retorten verlieren demnach ca. 15 Proz. Wasser beim Trocknen. Die lineare Schwindung beträgt 3,5 Proz. Die Porosität der Retorte beträgt 21,8 Vol.-Proz., das Raumgewicht ist $=2,0$. Eine aus St. Louis-Ton hergestellte Retorte stellt demnach ein äuBerst poröses Gefäß dar.

Ehe man die getrockneten Retorten an den Betrieb abgibt, kann man dieselben zuvor noch auf ibre Durchlässigkeit für Gase prüfen und speziell diejenigen Gefäße, welche ein in die Retorte gepumptes Quantum Luft allzu schnell entweichen Iassen, zurückstellen. Ich habe mir für diesen Zweck ein Prüfungsverfahren ausgearbeitet und verweise ich bezüglich desselben auf eine spätere Mitteilung.

Wenn völlig trocken, werden die Gefäße nach dem Verglühofen übergeführt.

II. Verhalten der Retorten im Feuer.

Die trockenen Retorten werden zunächst verglüht, dann bei Zinkofenhitze gargebrannt und endlich dem Betriebe übergeben, wobei sie während der Campagne andauernd den mannigfaltigsten Angriffen pyrochemischer und mechanischer Natur ausgesetzt bleiben. Dabei erleiden die GefäBe die verschiedenartigsten Zustandsänderungen, die man zum Teil zielbewubt herbeiführt und nützlichen Charakter haben, teils wider Willen eintreten, sich nicht vermeiden lassen, schädlich sind und schließlich die Retorte in unbrauchbaren Zustand versetzen.

Verglühen der Retorten. Durch das Verglühen bezweckt man, den Retorten eine gewisse mechanische Festigkeit zu verleihen, sie in einen Zustand überzuführen, in welchem sie ohne Gefahr transportiert und in den Zinkofen eingesetzt werden können. Dieses Ziel wird erreicht, wenn man die Ge- fäße nur so lange erhitzt, bis sie das Wasser vollständig abgegcben haben. Dann ist der Ton am unempfindlichsten gegen plötzliche Abkühlung, wie solche beim Hersusnehmen der Retorten aus dem Glühofen und beim Transporte eintritt, wie auch gegen eine plötzliche Überhitzung, wie sie statthat, wenn man die Röhre in den hocherhitzten Zinkofen einsetzt. Daß die Retorten in dem $\mathrm{Zu}$ stande, in welchem der Bindeton gerade das Konstitutionswasser verloren hat, die Eigenschaft besitzen, sowohl Kontraktion wie Expansion zu ertragen, ohne daß der Scherben durch das Entstehen von Rissen geschädigt wird, hat wohl seinen Grund darin, daB sich die den Scherben zusammensetzenden Bestandteile noch in derselben $z$ wangslosen Lage befinden, in welche sie durch die Verformung bez. durch das nachfolgende Schwinden beim Trocknen versetzt worden sind. Der die Sandkörner verkittende Bindeton besitzt jetzt die größte Porosität und hat seine Schwindung im Feuer noch nicht begonnen. Spannungen, wie sie zwischen Bindeton und Chamottesand dann eintreten, wenn ersterer durch höheres Erhitzen die Schwindung begonnen hat, existieren jetzt noch nicht. Sind aber einmal Spannungen dieser Art im Scherben vorbanden, so tritt bei plötzlichem Temperaturwechsel Bruch ein. Je nach den Umständen bilden sich sogenannte Haarrisse, oder aber es entstehen auf der Außenfläche des Scherbens mehr oder weniger tiefgehende Risse, welche bienenwabenartig aussehende Felder abgrenzen. Das Verglühen der Retorten hat daher mit Vorsicht zu geschehen und soll man sowohl totale wie lokale Überhitzung vermeiden, will man nicht die $\mathrm{Re}^{-}$ torten mehr oder weniger zur Schadhaftigkeit prädisponieren.

In Anbetracht der Größe der Gefäße und der verhältnismäßigen Unvollkommenheit der Heizapparate ist die Erfüllung dieser Aufgabe eine etwas schwierige.

Der folgende Versuch illustriert, in welcher Weise die Retorten beim Erhitzen das Wasser abgeben. Ein Bruchstück einer Retorte wurde im Glühofen mit den Retorten zusammen erhitzt und alle 2 Stunden in der Nähe des Versuchsstücks mit dem Pyrometer von Le Châtelier sowohl die Temperatur gemessen, wie der Gewichtsverlust durch Wiegen ermittelt. Dabei wurden die Zahlen der umstehenden Tabelle erhalten.

Das Retortenstück wog ursprünglich $233,3 \mathrm{~g}$, nach dem Verglühen nur noch $213,3 \mathrm{~g}$. Es verlor demnach beim Glühen $15,0 \mathrm{~g}$.

Man sieht, wie zuerst nur die Feuchtigkeit ausgetrieben wird. Zwischen $133-376^{\circ}$ 
entweicht etwas Wasser, das ron der beginnenden Verkokung der vorhandenen organischen Substanz von Limonit und Gips herrühren wird. Erst zwischen $376-660^{\circ} \mathrm{C}$. entweicht dann das Konstitutionswasser der Tonsubstanz, von da ab dürfte der Gewichtsverlust durch Ausbrennen von etwas organischer Substanz, Schwefel u. s.w. verursacht werden. Das Verglühen hat also wesentlich das Austreiben des Wassers zum $\mathrm{Z}_{\text {weck. Die Retortenmasse selbst wird durch }}$ das Brennen nicht verändert und befinden sich namentlich die FluBmittel noch auf primärer Lagerstätte, sie haben noch keine Wirkung ausgeübt.

\begin{tabular}{|c|c|c|c|c|}
\hline $\begin{array}{c}\text { Zeit der } \\
\text { Mestang u. } \\
\text { Wagugg } \\
\text { Uhr }\end{array}$ & $\begin{array}{c}\text { Gowleht } \\
8\end{array}$ & $\begin{array}{c}\text { Gewiohte- } \\
\text { abnahme } \\
\text { g }\end{array}$ & $\begin{array}{c}\text { Gewichtg- } \\
\text { abnuhme } \\
\text { auf Geramt } \\
\text { verluat } \\
\text { berechnet } \\
\text { Prox. }\end{array}$ & $\begin{array}{c}\text { Tempers- } \\
\text { tur } \\
\text { o } \mathrm{C} .\end{array}$ \\
\hline 2 & 233.3 & - & - & 14 \\
\hline 4 & 2320 & 1.3 & 8.7 & 149 \\
\hline 6 & 2316 & 0.4 & 2,6 & 149 \\
\hline 8 & 2314 & 0,2 & 1,3 & 149 \\
\hline 10 & 231,3 & 0,1 & 0,7 & 149 \\
\hline 12 & 231,3 & - & - & 116 \\
\hline 2 & 231,3 & -- & - & 133 \\
\hline 4 & 231,3 & - & - & 133 \\
\hline 6 & 230,5 & 0,8 & 5,3 & 262 \\
\hline 8 & 229,6 & 0,9 & 6,0 & 349 \\
\hline 10 & 229,5 & 0,1 & 0,7 & 402 \\
\hline 12 & 229,5 & - & - & 376 \\
\hline 2 & 228,5 & 1,0 & 6,7 & 481 \\
\hline 4 & 224.0 & 4.5 & 30.0 & 533 \\
\hline 6 & 219,8 & 4,2 & 28,0 & 627 \\
\hline 8 & 219,5 & 0,3 & 2,0 & 660 \\
\hline 10 & 219,2 & 0,3 & 2,0 & 715 \\
\hline 12 & 219,0 & 0,2 & 1,3 & 737 \\
\hline 2 & 218,6 & 0,4 & 2,7 & 770 \\
\hline 4 & 218,3 & 0,3 & 2,0 & 792 \\
\hline 6 & 218,3 & - & - & 781 \\
\hline
\end{tabular}

Die Operation des Verglühens wird in einem Flammofen, dessen Sohle vielfach durchbrochen ist, ausgeführt. Man stellt die Röhren in den über der Sohle befindlichen, durch ein Gewölbe abgeschlossenen Heizraum und befeuert den Ofen derart, daB die durch eine weite Öffnung in den Glühraum eindringende Flamme den Raum möglichst gleichmäBig erfüllt und durch viele Abzugsöffnungen allseitig dem Schornstein zugeführt wird. Nach 12-14-stündigem Erhitzen sind die Retorten so weit vorbereitet, daß sie in die Zinköfen übergeführt werden können.

Das Garbrennen im Zinkofen. Die modernen Zinköfen sind lange durch eine Mittelwand in 2 Hälften geteilte Flammöfen und stehen mit einer Generatorgasfeuerung in Verbindung. Der am einen Ende eintretende Gasstrom wird durch die Mittelwand gespalten, durchströmt beide Abteilungen und wird in denselben durch seitlich zugeführte Sekundärluft verbrannt. Da die Luftzufuhr an mehreren Stellen, welche gleiche Abstände von einander haben, erfolgt, so wird dadurch der Ofen beiderseitig in eine Anzahl von Verbrennungskammern - in Sektionen aufgeteilt. Die den beiden Abteilungen des Ofens entströmenden Verbrennungsgase vereinigen sich, kurz ebe sie in einen gemeinschaftlichen Schornstein eintreten. Die Retorten liegen in den einzelnen Kammern in einer Anzahl von Reihen übereinander, sodaß der allergrößte Teil der Oberfäche von den Verbrennungsgasen umspült wird. Die Röhren liegen mit ihrem hintersten Teile auf den Konsolen der Mittelwand auf, während ihr vorderer Teil auf Platten ruht. Sie sind nach vornehin etwas geneigt, damit sie bequemer beschickt bez. entleert werden können. Die Zahl der Röhren, welche ein solcher Ofen aufnehmen kann, schwankt je nach der Größe. Man hat Doppelöfen gebaut, die bis zu 1008 Retorten, auf jeder Seite 504, aufnehmen können.

In den einzelnen Teilen des Zinkofens herrscht zu der Zeit, während welcher die schadhaften Retorten dureh neue ersetzt werden, eine Temperatur, die zwischen 1200 bis $1350^{\circ} \mathrm{C}$. schwankt. Nachdem die zu erneuernde Röhre aus dem Ofen entfernt ist, geschieht das Einsetzen der verglühten Retorte in der Weise, $d a ß$ man sie mit einem Gestänge in den Ofen einschiebt und, nachdem sie in die richtige Lage gebracht worden ist, mit einer aus Ton und Chamottesand bestehenden, verhältnismäßig leicht zusammenbackenden Masse, die rings um die Röhren herum aufgetragen wird, in den Ofen einschließt.

Die Retorte verläBt den Glühofen mit einer Temperatur von 750 bis $780^{\circ} \mathrm{C}$. Sie kühlt sich während des Transportes etwas ab und erleidet beim Einsetzen in den Zinkofen eine plötzliche Temperatursteigerung um ca. $600^{\circ} \mathrm{C}$. Durch diese enorme Überhitzung, welche der Mantel der Retorte im Gegensatz ${ }^{2}$ ) zu den tiefer liegenden Partien erleidet, wird sich derselbe plötzlich auszudehnen versuchen und reibt dabei event. netzartig in jene durch mehr oder weniger tiefgehende Risse begrenzte Felder. Auch beim Auflegen von feuchter Masse um den Retortenmund herum können Risse entstehen, wodurch die Retorte an dieser Stelle gespalten wird.

$\mathrm{Da}$ die Retorte gegen das Ende einer Destillationsperiode in den Ofen eingesetzt wird, so erfährt sie zunächst cine weitere

2) Durch die Porosität des Scherbens wird dieser Gegensatz, wenn auch nicht viel, so doch etwas gemildert. 
Temperatursteigerung. Kurz vor und nach der Beschickung der Nachbarretorten tritt jeduch ein Sinken der Temperatur ein. Im Laufe der nun folgenden Periode erleidet die Retorte zum crsten Male die volle Erhitzung. Dabei wird sie gargebrannt und verlaufen im Scherben z. T. jetzt schon diejenigen Reaktionen, welche ich früher ausführlich beschrieben habe ${ }^{3}$ ). Die Tonsubstanz schwindet und die FluBmittel beginnen ihre Tätigkeit, und zwar im hinteren Teile der Retorte, wo die Hitze am gröBten ist, mehr als vorne, wo die Retorte in der Wand liegt, am Mantel mehr, als an den der Innenfläche zugekehrten Teilen. Die Röhre zieht sich infolge der pyrochemischen Vorgänge mehr und mehr zusammen und ist diese Schwindung wiederum an den höcht erhitzten Stellen am bedeutendsten.

Entnimmt man eine frisch eingesetzte Retorte nach 24-stündigem Erhitzen dem Ofen, so bemerkt man an derselben bereits Veränderungen. Sowohl der Retortenmantel als auch die auf demselben auftretenden Risse haben sich infolge der Überschüttung mit Flugstaub braungelb gefärbt und ist letzterer auch in die Risse eingedrungen. Die Oberfläche der Retorte besitzt jetzt schon eine äuBerst dünne Schicht einer Glasur. Auch zeigt der Mantel an vielen Stellen kleine Löcher. Ähnliche Höhlungen bemerkt man im Innern des Scherbens. Sie sind zum Teil noch mit einer bräunlichen Masse ausgefüllt, der größere Teil davon hat sich jedoch in den weiBlichen Scherben hineingezogen und bildet, um das Loch herum, einen braunen bez. bräunlichgelben Hof. Diese Löcher rübren offenbar von Eisenoxyd bez. auch von Schwefelkies, Gips, Kalkspat u. s. w. her, welche nach dem Abgeben ron $\mathrm{S}$ bez. $\mathrm{CO}_{2}$ als Oxyde aufschließend auf die Tonsubstanz einwirken und damit leicht schmelzbare Silikate bilden, die allmählich, unter Zurücklassung einer Höhlung, von dem porösen Scherben aufgesaugt werden und demselben seine Farbe verleihen. Dieser Proze $B$ hat sich nach 24-stündigem Erhitzen am Mantel - also da wo die Hitze am gröBten ist schon nahezu vollzogen, während die im Innern des Scherbens befindlichen Löcher ganz oder z. T. noch mit Eisenoxyd u. s. w. ausgefüllt sind.

Ich habe früher mitgeteilt ${ }^{4}$, wie sich die Retortenmasse in den einzelnen Stadien des Brennens in Bezug auf Porosität, Schwindung u. s. w. verhält. In verglühtem Zustande besitzt der Scherben eine Porosität von

3) Vergl. Zeitschr. f. angew. Chem. 1903 S. 151.
4) Vgl. Zeitschr. f. angew. Chem., letzte Abhandlung.

Ch. 1.903
28,0 Vol.-Proz. und einen Gewichtsraum von 52,0 $\mathrm{cm}$ pro $100 \mathrm{~g}$ Scherben. Gargebrannt dagegen eine Porosität von 24,5 Vol.-Proz. und einen Gewichtsraum von $50 \mathrm{ccm}$. Durch das Garbrennen verringert sich demnach die Porosität um 3,5 Vol.-Proz. Der Nachschwund beträgt $2 \mathrm{ccm}$ pro $100 \mathrm{~g}$ Scherben. Das Erhitzen auf höhere Temperatur erhöht auch die Festigkeit der Röhre. Man darf sich eben den hocherhitzten Ton nicht mehr als spröde Masse - wie wir es zu tun gewöhnt sind - vorstellen, sondern als eine mehr oder weniger elastische Substanz, welche gegen StoB und Schlag wenig mehr empfindlich ist. Man wird nicht irre gehen, wenn man den im St. Louis-Ton enthaltenen FluBmitteln die Eigenschaft zuschiebt, diesen $\mathrm{Zu}$ stand zu veranlassen, bez. den aus Tonsubstanz, Kieselsäure und den FluBmitteln sich bildenden Doppelsilikaten, deren Natur wir zwar nicht kennen, welche aber den Scherben in homogener Weise durchdringen und sich bei den in Frage kommenden Temperaturen in einer Art halbfestem Zustande befinden, ähnlich dem, in welchem sich das Eisen schweißen läBt.

Verhalten der Retorte während dem Gebrauche. Während der Lebensdauer wird die Retorte täglich einmal mit der feuchten Kohleerzmischung aufgefüllt. Das aus der Reduktion von Zinkoxyd hervorgehende Metall kondensiert und sammelt sich zum gröBten Teile in der in die Retorte gesteckten Vorlage, welche von Zeit zu Zeit entleert wird. Nach dem Abtreiben der Hauptmenge des Zinks kratzt man den in der Retorte verbliebenen Rickstand "die Asche" heraus und beschickt von neuem. Dieses Beschicken und Entleeren wiederholt sich täglich, bis die Röhre unbrauchbar geworden ist und infolge eines erlittenen Schadens, in der Regel wegen einem Längs- oder Querriß herausgenommen werden muß, was nach 2 bis 3 Monaten der Fall ist.

Während dem Gebrauche ist das Gefäß den mannigfachsten pyrochemischen Einflüssen ausgesetzt, welche sich namentlich in dreierlei Richtungen geltend machen. Der aus den Aschenbestandteilen der bituminösen Kohle hervorgehende, im wesentlichen aus Quarzsand, Tonsubstanz, Eisenoxyd bez. Schwefeleisen, Kalk, Magnesia und Alkalien bestehende, mehlfeine Flugstaub, von welchem die die Retorte umspülenden hocherhitzten Verbrennungsgase geschwängert sind, greift die Tonsubstanz der porösen GefäBe an unter Bildung einer Glasur, welche sich im Laufe der Zeit mehr und mehr verdickt und das Gefä日 total einhüllt. 
Gleichzeitig wird durch dis im Innern der Retorte erzeugten Zinkdāmpfe dic Tonsubstanz vollständig in Zinkaluminat - Zinkspinel1 $\left.{ }^{5}\right)-$ gemäß:

$$
\begin{gathered}
\mathrm{Al}_{3} \mathrm{O}_{3} \cdot 2 \mathrm{SiO}_{2}+\mathrm{Zn}+\mathrm{CO}_{2}= \\
\mathrm{Al}_{2} \mathrm{O}_{3} \cdot \mathrm{ZnO}+\mathrm{CO}+2 \mathrm{SiO}_{2}
\end{gathered}
$$

umgewandelt, während die zuvor an die Tonsubstanz gebundene Kieselsäure, sowie der im Ton vorhandene Quarz zum Teil in Tridymit übergehen.

Der Zinkspinell entsteht namentlich in der ersten Zeit der Benutzung der GefäBe, solange die an sich vollständig poröse $\mathrm{Re}$ torte noch keine Glasur auf der den Feuergasen dargebotenen Seite gebildet hat und Zinkdämpfe sowohl wie Verbrennungsgase durch die Retorte hindurchdiffundieren können.

Ich habe Analysen ${ }^{6}$ ) von im Gebrauche gewesenen Zinkretorten ausgeführt und fand, daB die Zinkaufnahme im wesentlichen in den ersten 8 Tagen der Benutzung des Ge-

\begin{tabular}{|c|c|c|c|}
\hline In Gebradohe & Gewiaht & Zink-Gohalt & or Retorte \\
\hline Tego & Pfd. & Proz. & Pfd. \\
\hline $\begin{array}{r}8 \\
20 \\
135\end{array}$ & $\begin{array}{l}163,00 \\
171,25 \\
176,50\end{array}$ & $\begin{array}{l}13,50 \\
12,75 \\
15,15\end{array}$ & $\begin{array}{l}22,00 \\
21,83 \\
26,78\end{array}$ \\
\hline
\end{tabular}
fäßBes erfolgt:

Da der gebrannte St. Louis-Ton 39,26 Proc. $\mathrm{Al}_{2} \mathrm{O}_{3}$ enthält und das Durchschnittsgewicht einer Retorte im gargebrannten Zustande nach meinen Trmittelungen 146,75 Pfd. beträgt, so enthält eine Retorte $57,61 \mathrm{Pfd} . \mathrm{Al}_{2} \mathrm{O}_{3}$. Diese würden zur Zinkspinellbildung 45,70 Pfd $\mathrm{ZnO}$ benötigen. Derartig hohe Zinkoxydaufnahmen erleiden jedoch, wie aus der Tabelle ersichtlich ist, die Retorten nicht, da eben die Tonerde nur zum Teil in Zinkspinell übergeht. Man hat es also schon nach einigen Tagen nicht mehr mit einem aus feuerfestem Tone bestehenden GefäBe zu tun, sondern mit einem solchen, dessen Scherben zum gröBten Teile aus Zinkspinell und Tridymit besteht.

Bei welchen Temperaturen die Bildung des Zinkspinells erfolgt, habe ich nicht ermitteln können. Ich habe aber gefunden, dab die Reduktionstemperatur des Zinkoxyds bei ca. $1000^{\circ} \mathrm{C}$. liegt, also $60^{\circ} \mathrm{C}$. höher als der Siedepunkt des Zinks ${ }^{7}$ ),

5) Vgl. auch A. Stelzner und H. Schulze (Wagners Jahresbericht 1881, S. 109) und V. Stege r (Wagners Jahresbericht 1887, S. 458).

G) Vgl. O. Mülhaeuser: Analyse zinkspinellhaltiger Scherben. Ztschr. f. anorg. Chemie 1902. S. 1242.

7) Roberts Austen und Berthelot ermittelten den Siedepunkt des Zinks mit dem Pyrometer von Le Châtelier bei $940^{\circ} \mathrm{C}$. (Compt. rend. 131 , S. 380 und Journal of the Society of Chem. Industry Oct. 3, 1900). und glauhe annohmen zu clürfen, daß sich der Zinkspinell namentlich bei 'Temperaturen uiber $1000^{\circ} \mathrm{C}$. bildet.

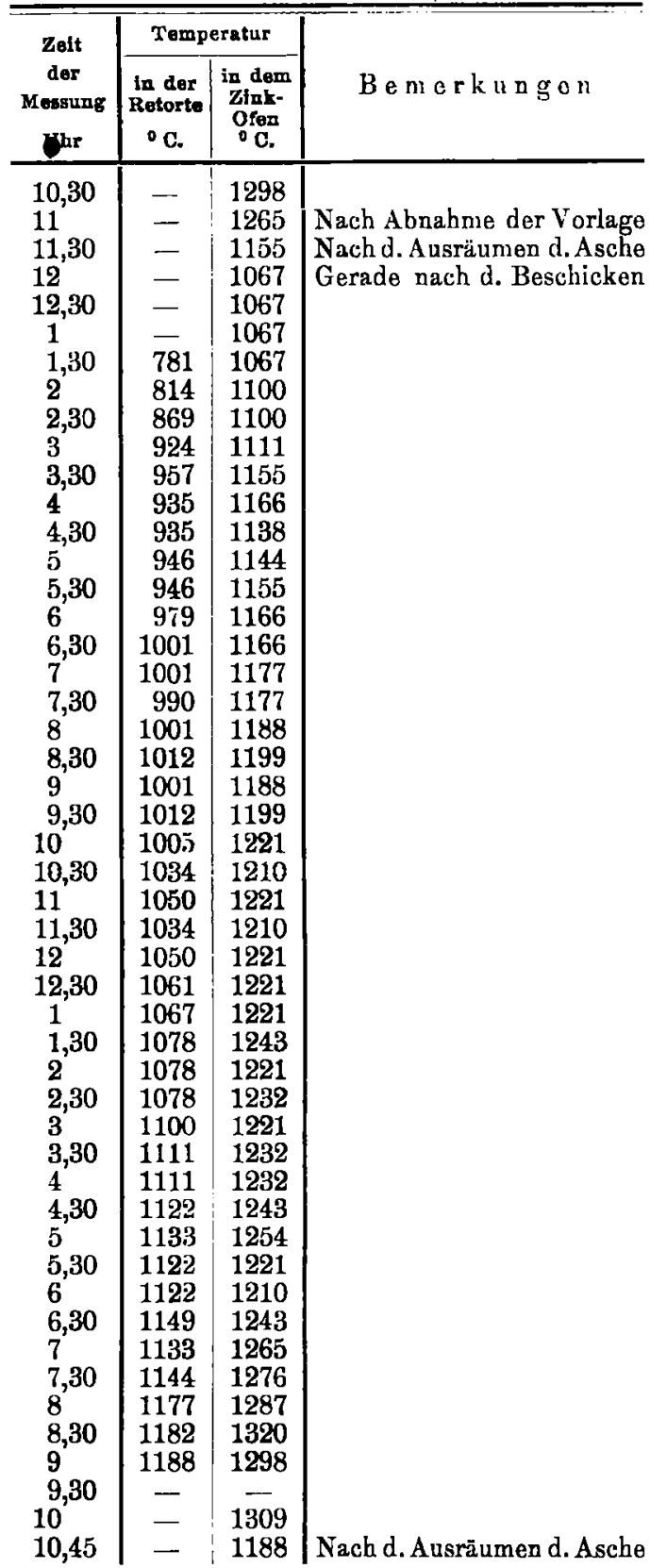

Als weiterer die Struktur des Gefäbes alterierender Einflub tritt die aus der Asche der Ingredienzien event. aussaigernde dünnflüssige Schlacke auf, welche unter Umständen in die Unterseite der Retorte eindringt und dieselbe an den der Einwirkung ausgesetzten Stellen in eine wenig poröse Masse umwandelt. Was die Natur dieser Schlacken betrifft, so ist darüber im allgemeinen wenig bekannt. Man weib, daß Tonerdesilikate an und für sich streng- 
flüssig sind, so lange sie keine Gelegenheit finden, mit Kalk- bez. EisenoxydulSilikaten leichtflüssige Doppelsilikate zu bilden, und gattiert daher, wenn es die Verhältnisse gestatten, in der Weise, daß man entweder eine rein kieselige oder eine rein basische Gattierung erhält. Derartig gattierte

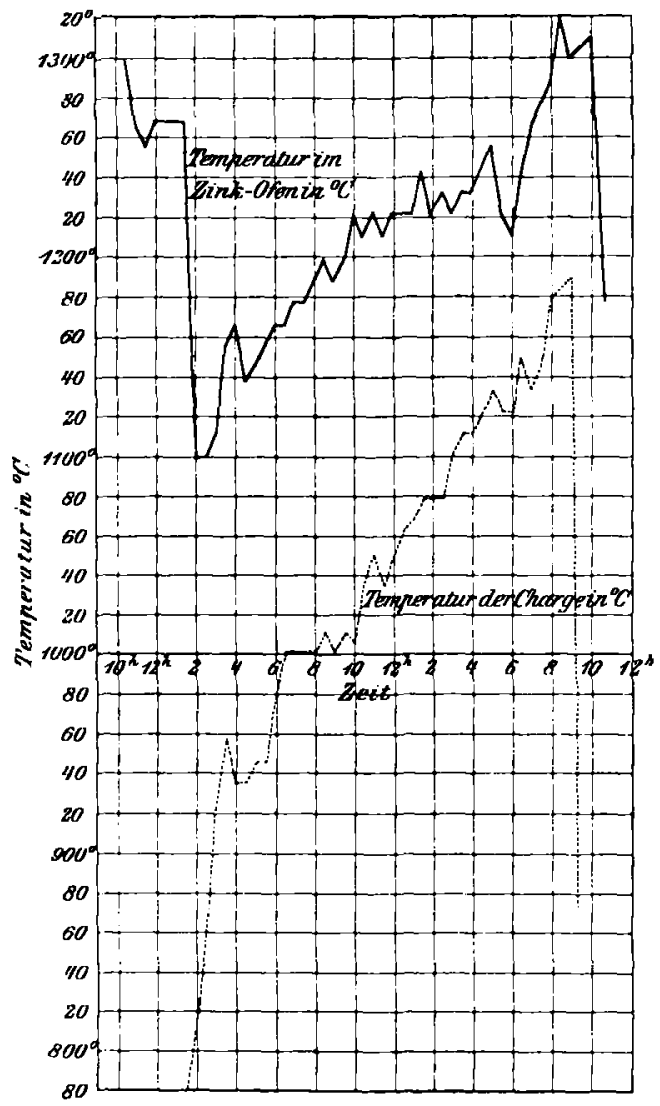

Erze lassen trockene die Retorten nicht angreifende Aschen erwarten. Da aber die Verhältnisse diese Art der Gattierung in den meisten Fällen nicht gestatten, so setzt man eben die Mischung so zusammen, dab bei der zum Abtreiben des Zinks schlieblich nötig werdenden hohen Temperatur eine möglichst schwer schmelzbare Schlacke entsteht. Aschen, welche die Schlacke annähernd in diesem $\mathrm{Zu}$ stande enthalten, bekommt man, wenn große Mengen von Kalk, bez. von Kalk und Magnesia oder von Tonerde und nur geringe Mengen von Kieselsäure oder umgekehrt größere Mengen von Kieselsäure gegenüber geringen Mengen von Basen in der Gattierung vorhanden sind.

Was nun die Temperaturgrenzen betrifft, innerhalb welcher sich diese pyrochemischen Umsetzungen vollziehen, so ergeben sich dieselben aus der Tabelle, in welcher ich eine Reihe von Messungen verzeichnet habe, welche während einer Destillierperiode innerhalb der Retorte - inmitten der Asche - und im Zinkofen dicht bei der Retorte ausgeführt worden sind. (Vergl. das Diagramm.)

Man sieht aus diesen Zahlen, wie mit der Zeit, wenn das Zink mehr und mehr abgetrieben wird, auch die Temperatur der Asche sich mehr und mehr derjenigen des Zinkofens nähert, um sie schließlich unter Umständen zu erreichen. Hat die Asche die Temperatur des Ofens annähernd oderganz angenommen, so ist auch die Gefahr vorhanden, daB Schlacke aus der Asche ausschmilzt und, je nachdem die Verhältnisse liegen, mehr oder weniger leicht von dem Scherben der Retorte aufgesaugt wird und lokale Veränderungen verursacht.

Nachdem ich im Vorhergehenden die chemischen Einflüsse, denen eine Retorte im Feuer ausgesetzt ist, ganz im allgemeinen dargelegt habe, möchte ich noch an einem speziellen Beispiele zeigen, in welcher Weise eine Retorte, welche während 135 Tagen im Feuer gelegen und zur Zinkdestillation gedient hatte, in ihren einzolnen Teilen verändert wurde, und gebe ich daher in der folgenden Tabelle einerseits die Analysen der aufeinander einwirkenden Ingredienzien: also vom Ton, vom Flugstaube und Erz und andererseits solche von den aus der Einwirkung hervorgehenden Produkten: von der Glasur, dem Scherben u. s. w.

\begin{tabular}{|c|c|c|c|c|c|c|c|c|c|c|c|c|c|}
\hline Zusammensetzi & $\left|\begin{array}{l}\mathrm{Si}_{2} \\
\text { Proz. }\end{array}\right|$ & $\left|\begin{array}{l}\mathrm{Al}_{2} \mathrm{O}_{3} \\
\text { Proz. }\end{array}\right|$ & $\begin{array}{l}\text { FeO } \\
\text { Proz. }\end{array}$ & $\begin{array}{l}\text { C: } 0 \\
\text { Prox. }\end{array}$ & $\begin{array}{l}\text { Mg O } \\
\text { Proz. }\end{array}$ & $\begin{array}{l}K_{2} \mathbf{O} \\
\text { Proz. }\end{array}$ & $\begin{array}{l}\mathrm{N} 2, \mathrm{O} \\
\text { Proz. }\end{array}$ & $\begin{array}{l}\text { Pb O } \\
\text { Proz. }\end{array}$ & $\left|\begin{array}{l}\text { Zno } \\
\text { Proz. }\end{array}\right|$ & $\begin{array}{l}\text { Cr } \mathrm{O} \\
\text { Proz. }\end{array}$ & $\begin{array}{c}\mathbf{8} \\
\text { Proz. }\end{array}$ & $\begin{array}{l}\text { Cd O } \\
\text { Proz. }\end{array}$ & Bem \\
\hline 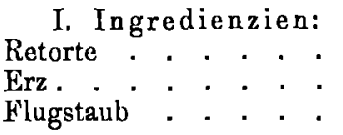 & $\begin{array}{r}56,08 \\
7,00 \\
9,48\end{array}$ & $\begin{array}{r}39,26 \\
2,22 \\
2,38\end{array}$ & $\begin{array}{l}2,48 \\
1,15 \\
2,86\end{array}$ & $\begin{array}{l}0,78 \\
0,80 \\
0,36\end{array}$ & $\begin{array}{l}0,51 \\
0,07 \\
0,05\end{array}$ & $\frac{0,07}{0,27}$ & $\mid \begin{array}{c}0,19 \\
\overline{0.35}\end{array}$ & $\begin{array}{c}\overline{0}, \overline{37} \\
-\end{array}$ & $\begin{array}{c}\overline{86,84} \\
12,40\end{array}$ & $\overline{0,08}$ & $\begin{array}{l}\overrightarrow{1,17} \\
8,56\end{array}$ & $\overline{0,36}$ & C nicht bestimmt \\
\hline 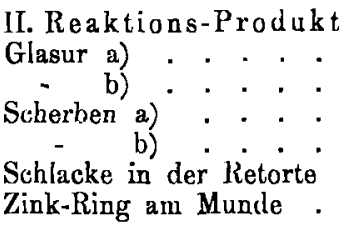 & $\begin{array}{r}50,26 \\
54,98 \\
53,70 \\
44,68 \\
66,94 \\
5,90\end{array}$ & \begin{tabular}{|r|}
31,84 \\
32,78 \\
37,03 \\
32,52 \\
13,66 \\
3,85
\end{tabular} & $\begin{array}{r}8,85 \\
5,40 \\
3,48 \\
3,24 \\
11,12 \\
1,09\end{array}$ & $\begin{array}{l}1,40 \\
1,96 \\
0,60 \\
0,10 \\
4,34 \\
0,20\end{array}$ & $\begin{array}{c}0,36 \\
0,64 \\
- \\
\overline{1.30} \\
-\end{array}$ & $\begin{array}{c}1,09 \\
0,51 \\
0,49 \\
0,11 \\
0,52 \\
-\end{array}$ & $\begin{array}{c}1,68 \\
1,81 \\
0,54 \\
0,20 \\
0,48 \\
-\end{array}$ & $\begin{array}{c}0,28 \\
0,04 \\
- \\
- \\
\overline{1,95}\end{array}$ & $\begin{array}{r}3,20 \\
0,22 \\
4,12 \\
19,10 \\
3,42 \\
88,60\end{array}$ & $\begin{array}{c}\overline{0,02} \\
- \\
- \\
0,16\end{array}$ & $\begin{array}{c}- \\
- \\
\overline{1,29} \\
- \\
-\end{array}$ & $\begin{array}{l}- \\
- \\
- \\
\overline{-} \\
\overline{0,05}\end{array}$ & $\begin{array}{l}\text { glattes Aussehen } \\
\text { blasiges Aussehen } \\
\text { vom Retorten-Munc } \\
\text { vom Boden der Retor }\end{array}$ \\
\hline
\end{tabular}


III. Ursachen der Gefäßzerstörung.

So wie die Retorte aus dem Zinkofen herauskommt, stellt dieselbe einen weißglühenden Zylinder dar, der sich selbst durch den Schlag eines schweren Hammers nur schwierig zertrümmern lassen würde. Mit der Eirniedrigung der Temperatur nimmt jedoch die Festigkeit $a b$ und beim Erkalten zerspringt die Röhre zumeist und sind es namentlich Längs- und Querrisse, welche den Zerfall herbeiführen.

Die erkalteten GefäBe besitzen eine je nach dem Alter mehr oder weniger stark entwickelte Glasur. Die Farbe des Glases ist tief schwarz, zuweilen mit einem Stich ins Grüne oder Braune, je nach dem mehr reduzierenden oder oxydierenden Charakter der die Röhre umspielenden Verbrennungsgase. Der Scherben ist graublau, dunkelblau, graugrün, graugelb oder grüngelb gefärbt.

Wie früher mitgeteilt wurde, werden die Gefäße in den. meisten Fällen wegen einem Längs- oder Querriß aus dem Ofen entfernt. Es sind das aber nicht die einzigen Schäden, welche die Retorten erkennen lassen. Beim Zerschlagen bemerkt man namentlich a uf dem Mantel der Röhre noch die Spuren jener früher erwähnten netzartig verzweigten, beim Einsetzen der Retorten entstandenen Risse, die sogenannten "Mantelrisse". Sie sind zwar von Glasur bedeckt und offenbar bez. scheinbar verheilt. Bei näherer Betrachtung entdeckt man aber, daB sāmtliche Risse Wege darstellen, auf denen die auf der Mantelfläche gebildete Glasur ins Innere des Scherbens gelangt. Überall da, wo sich ein Riß vorfindet, ist die Tonmasse zu beiden Seiten des Risses bienenwabig ausgefressen und ist derselbe je nach der Tiefe, der Weite der Spalte und Qualität der eingedrungenen Schlacke der Herd einer mehr oder weniger grolen Zerstörung geworden. Dagegen habe ich ein tieferes Eindringen der Schlacke bez. der Glasur in den Scherben unterhalb der ersteren, ein Aufsaugen derselben durch die Poren nie beobachten können. Das Eindringen findet höchstens einige Millimeter tief statt und pflegt man eben diesen Teil der Retorte als Glasur zu bezeichnen, da sowohl der Flugstaub wie die unmittelbar darunter liegende Schicht des Scherbens infolge der Flugstaubbeschüttung einheitlich verschlackt sind. Je nach dem
Alter der Retorte, der Lage gegenüber den Winddüsen bez. der Lage im Ofen überhaupt ist die Glasur mehr oder weniger stark entwickelt. Wird die Schlacke aus irgend einem Grunde, sei es infolge allzustarker Flugstaubbeschüttung oder wegen zu intensiver Hitze, leichtflüssig, so fließt sie ab. Über den Rissen, namentlich an solchen Stellen, wo zwei Spalten zusammenstoßen, bemerkt man häufig kraterförmige Erhöhungen, welche dartun, dal hier Gasausbrüche stattgefunden haben.

Auf der Innenseite der Röhren bemerkt man manchmal Risse ähnlicher Art, von derselben netz- bez. schachbrettartigen Zeichnung und demselben inneren Zusammeuhang. Speziell auf der unteren Seite der Retorte sind die Risse mit Schlacke bedeckt, im oberen Teile dagegen deutlich sichtbar. Wodurch diese Risse verursacht werden, habe ich nicht feststellen können. Es ist möglich, daß sie schon beim Einsetzen der Retorten entstehen, vielleicht aber auch erst später beim Einwerfen der nassen Beschickung in die hocherhitzte Röhre. Sind diese Risse tiefgehend und klaffend, so füllen sie sich bald mit dünnflüssigen Doppelsilicaten an und indem letztere in die von den Rissen begrenzten Felder des Scherbens, von allen Seiten einwandernd, sich mit der Tonmasse verbinden, entsteht an den dieser Einwirkung ausgesetzten Stellen der Retorte ein steinzeugartig verdichteter Scherben, welcher einen ganz anderen AusdehnungsKoëffizienten besitzt als die nachbarliche nicht beeinflubte porōse Masse. Der Scherben wird daher an solchen Stellen, wo die dichte Masse mit der porösen zusammenstöbt, infolge der verschiedenen Ausdehnung der Teile leicht brechen. Man kann dieselbe Erscheinung auch in der Nähe des Mundes einer Retorte beobachten, wo äbnliche Unterschiede im Charakter benachbarter Teile auftreten können. Je nachdem dann die Versinterung mehr in der Längs- oder Querrichtung fortgeschritten ist, wird auch der $\mathrm{Ri \beta}$ bei eintretender Gelegenheit in der einen oder anderen Richtung verlaufen.

Wie sehr die Dichtigkeit einer Retorte an verschiedenen Stellen variieren kann, geht aus Bestimmungen hervor, die ich mit einigen Scherben ausgeführt habe, die verschiedenen Teilen einer Retorte entnommen worden waren. (Vgl. die Tabelle.)

\begin{tabular}{|c|c|c|c|c|c|c|c|}
\hline & $\begin{array}{c}\text { Gewlcht } \\
\mathrm{g} \\
\end{array}$ & $\begin{array}{c}\text { Sehorben } \\
\text { plua } \\
\text { Watier } \\
8 \\
\end{array}$ & $\begin{array}{c}\text { Ab- } \\
\text { sorbiertes } \\
\text { Wasser } \\
\text { g }\end{array}$ & $\begin{array}{c}\text { Volum } \\
\text { ccm }\end{array}$ & $\begin{array}{l}\text { Porositat } \\
\text { Vol.-Proz. }\end{array}$ & $\frac{100 \cdot \nabla}{\mathrm{g}}$ & Spez. Gow. \\
\hline $\begin{array}{l}\text { Stück vom runden Boden } \\
\text { Stück der Röhre von oben } \\
\text { Stück der Röhre von unten }\end{array}$ & $\begin{array}{l}161,35 \\
116,75 \\
124,80\end{array}$ & $\begin{array}{l}176,10 \\
129,40 \\
132,70\end{array}$ & $\begin{array}{r}14,75 \\
12,65 \\
7,90\end{array}$ & $\begin{array}{l}70,0 \\
58,3 \\
58,5\end{array}$ & $\begin{array}{l}21,1 \\
21,7 \\
14,8\end{array}$ & $\begin{array}{l}43,4 \\
50,0 \\
42,9\end{array}$ & $\begin{array}{l}2,93 \\
2,48 \\
2,75\end{array}$ \\
\hline
\end{tabular}


An frisch gargebrannten Retorten bez. an solchen, die noch nicht allzuoft zur Zinkdestillation benutzt worden sind, bemerkt man solche Unterschiede nicht, der Scherben solcher Retorten ist überall gleich dicht, an älteren Retorten dagegen kann man sehr häufig zwischen einer porösen oberen Hälfte und einer dichteren mehr oder weniger zusammengefritteten bez. steinzeugartig veränderten unteren Hälfte unterscheiden. Es kommen aber auch sehr alte Retorten vor, welche durchaus ihren porösen Charakter erhalten haben.

Eine weitere Art von Rissen sind die "Mundrisse", durch welche der Mund der Retorte ganz oder zum Teil durchgespalten wird. Sie entstehen zu allen Zeiten, entweder aus den schon entwickelten Gründen oder auch aus anderen Ursachen. Schon beim Einsetzen erfährt der Mund der Retorte, soweit er in die Vorderwand des Ofens zu liegen kommt, durch das Auflegen nasser Tonmasse eine bedeutende lokale Abkühlung, während der Rest der Retorte im Gegenteil der denkbar stärksten Erhitzung ausgesetzt wird. Auch später besitzt der Mund, da der Scherben ein schlechter Wärmeleiter ist, immer eine niedrigere Temperatur als die sich daran anschlieBenden Teile der Retorte. Letztere schwindet daher gerade an dieser Stelle weniger als an den anderen tiefer im Ofen liegenden Teilen. Es entstehen deshalb Spannungsdifferenzen, welche event. zur RiBbildung führen. Auch mechanische Einflüsse, wie das oft gewaitsame Ausbrechen der Vorlagen, das Abstoßen des sich im Retortenmunde bildenden Zinkwulstes, lassen häufg Risse zurück. Im allgemeinen sieht man, wie der in konstruktiver Hinsicht schwächste Teil der Retorte, der Mund, Kingriffe der gewaltigsten Art erfährt, und ist es nicht zu verwundern, daß keine einzige aus der Campagne hervorgehende Retorte an der fraglichen Stelle intakt geblieben ist. Die Mundrisse füllen sich häufig mit weißen und gelblich gefärbten Sublimaten an, welche der Hauptsache nach aus Zinkoxyd bestehen. Öfers erfolgt von der mit Zinkoxyd, manchmal auch mit Schlacke ausgefūllten Spalte aus plötzlich die Bildung eines oft einen Meter langen Längsrisses.

Löcher, welche durch die Wand hindurchgehen, entstehen nach meinen Beobachtungnen nur, wenn etwa zufällig Eisenteile in die Masse gelangen oder wenn im Erze vorhandene Eisenerze, besonderer Umstände halber, sich zu sammeln Gelegenheit finden. Dann frilt sich das Eisen an der Stelle, wo die von dünnflüssiger Schlacke umgebene Eisenmasse aufliegt, durch die Wand hindurch und bildet ein rundes Loch.
Außerdem bemerkt man öfter innerhalb des Scherbens kleine Löcher von der GröBe eines Hirsekorns bez. auch einer Erbse, welche vom Ausbrennen von Fremdkörpern herrühren und für die Haltbarlicit des $\mathrm{Ge}$ fäßes bedeutungslos sind.

\section{Zustand der Retorte} während des Gebrauchs.

Im Zinkofen geht demnach die Retorte durch drei Phasen der Umwandlung hindurch. Im ersten Stadium wird sie mehroder weniger gargebrannt, sie schwindet und paßt sich den im Ofen herrschenden Temperaturverbältnissen an. Im zweiten Stadium sättigt sie sich mit Zinkoxyd, „nimmt" - wie der Zinkhüttenmann sagt - „ibre Nahrung auf ${ }^{u}$, überzieht sich mit einer Glasur und schrumpft noch etwas ein. Im dritten Stadium endlich verändert sie sich namentlich noch durch die Einwanderung von Flubitteln in den Scherben an einzelnen Stellen. Die Mantelrisse erbält die Retorte meist schon beim Einsetzen in den Zinkofen. Die Mundrisse entstehen zu allen Zeiten während der Campagne, ebenso die Innenflächenrisse, welche hōchst wahrscheinlich beim Einwerfen der nassen, kalten Beschickung ihren Ursprung nehmen und Wege darstellen, durch welche die event. aus der Asche aussaigernde Schlacke in den Scherben der Retorte eindringt, um sie an den besonders dazu prädestinierten Stellen steinzeugartig zu verfritten bez. zu versintern, worauf dann augenscheinlich die Zerstörung des Gefäßes durch einen Längs- oder QuerriB erfolgt.

Eine Zinkretorte stellt demnach ein mehr oder weniger durch Risse mannigfaltiger Art geschädigtes, poröses GefäB dar, das der Hauptsache nacb aus Tonsubstanz, Zinkspinell und Quarz bez. Tridymit besteht. Sie ist von den ursprūnglich im Tone in geringen Mengen vorhandenen FluBmitteln gleichmäBig durchtrānkt, hat aber außerdem durch die Einwanderung von Flußmitteln von aulerhalb, lokale Verschlackung erlitten, so $\mathrm{da} \beta$ das Gefä $B$ an einzelnen Stellen mehr oder weniger stark steinzeugartig verändert ist, wāhrend es der Hauptsache nach seinen porösen Charakter beibehalten hat. Das Gefäb ist in der Zinkofenhitze mechanischen Einwirkungen gegenüber äußerst widerstandsfähig, was daher kommt, daB der im kalten Zustande an und für sich spröde Scherben weißglühend eine große Zähigkeit besitzt. 
V. Wertung einer Retorte.

Der Grad der Durchlässigkeit für Zinkdämpfe hängt von der Art (Mantel- und Innenflächenrisse) und Zabl der Risse, außerdem von der Dicke und Zähflüssigkeit der Glasur ab. Je größer die Zahl der Risse, je tiefer dieselben in die Wand eindringen, je dünner die darüber liegende Glasur ist, desto weniger Widerstand wird dem Durchgang der Zinkdämpfe dargeboten, desto leichter durchdringen letztere die poröse Wandung, desto größer ist daher auch der Verlust durch Verflüchtigung. Der Widerstand, den der Scherben dem Durchgang der Dämpfe durch die Wandung hindurch entgegensetzt, bez. die Beschaffenheit der letzteren bildet neben der Dauerhaftigkeit des Gefäßes im allgemeinen (Lebensdauer) das wichtigste Moment bei der Bewertung einer Retorte, da dadurch die Ausbeute an Metall in hohem Maße beeinflubt wird.

\section{Arbeitsresultate.}

Ich habe in sorgfältigster Weise an einigen Retorten die Verluste an Zink. welche 1. durch Absorption des Metalls rom porösen hochtonigen Scherben, also vorherrschend durch Zinkspinellbildung, 2. durch Verflüchtigung durch die Wandung hindurch, 3. durch das Entweichen der Dämpfe aus der Vorlage und 4. durch unvollständiges Abtreiben der Asche entstehen, festgestellt, um ein möglichst genaues Bild von der Bedeutung dieser Verluste zu gewinnen, und teile an dieser Stelle die Resultate einer Untersuchung mit, die ich während einer einwöchentlichen Versuchsperiode mit einer neu eingesetzten Retorte erhalten habe.

Aus den in der Tabelle verzeichneten Zahlen ist zu entnehmen, in welcher Weise sich der Fehlbetrag an Zink auf die einzelnen Verlustquellen verteilt bez. welche Ausbeuten an Metall die Retorte während der ersten Zeit des Betriebs - bis zum Eintritt normaler Arbeitsverhältnisse bei einem Metallverlust von ca. 8 Proz. - zu erhalten gestattete.
Die Zahl der gegenwärtig nuf den nordamerikanischen Zinkhütten in Betrieb befindlichen Retorten dürfte 50000 betragen, wovon entfallen:

auf den Südwesten (Kansas u. Missouri) ca. 34000 auf Illinois . . . . . . . . . . - 12000 auf den Osten und Süden (Pennsyl-

vania, Indiana, Virginia) . . . . - 4000 .

\section{Bürette mit automatischer Einstellung des Nullpanktes und Entleerung durch direktes ZarückflieBen der nicht ver- brauchten Flüssigkeit.} Von Dr. C. Zahn.

Bei dieser Būrette findet das Prinzip des Hebers zur automatischen Finstellung in der Weise Verwendung, daß, wie Fig. 1 zeigt, ein an ihrem oberen Ende zu einer Kapillare ausgezogenes Rohr $d$ sich vor dem unteren Bürettenverschluß $f$ abzweigt und oben in die zu einer Kugel erweiterte und mit einem Entlüftungsrohr versehene Bürette wieder einmündet. Durch diese Anordnung wird erreicht, daß nach Füllung der Bürette bis zu der kugelförmigen Erweiterung (durch Einblasen von Luft in das Gefäß $H$ mittels Gummigebläses) die Titerflüssigkeit, wenn man die komprimierte Luft durch ein Ventil wieder entweichen läßt, durch die Kapillare $d$ automatisch bis zum Nullpunkt der Bürette abgehebert wird. Zweckmäßig geschieht die Füllung des oberen Teiles der Bürette nach Scbließen des Hahnes $f$ nur durch die Kapillare $d$. Die nicht verbrauchte Flüssigkeit kann durch Ōffnen des Hahnes $f$ in das Vorratsgefäß $H$ zurũckgelassen werden.

Der Vorzug des Apparates besteht in schneller Füllung, stets präziser Einstellung des Nullpunktes, sparsamem Verbrauch der Titerflüssigkeit und Schutz derselben gegen ăußere Einflüsse, da sie bis auf das bedeckte Entlüftungsrohr $c$ von der Außenluft abgeschlossen bleibt.

Auch für Flüssigkeitsmengen von $25 \mathrm{ccm}$ und weniger können derartige Apparate unter Fortfall der Klemme $k$ ausgeführt werden.

Eine weitore Verwendung des angegebenen Prinzips für Vollpipetten veranschaulicht Fig. 2, wo sich an Stelle der Bürette eine Pipette befindet. Dieselbe ist nach unten bei $e$ verschlossen,

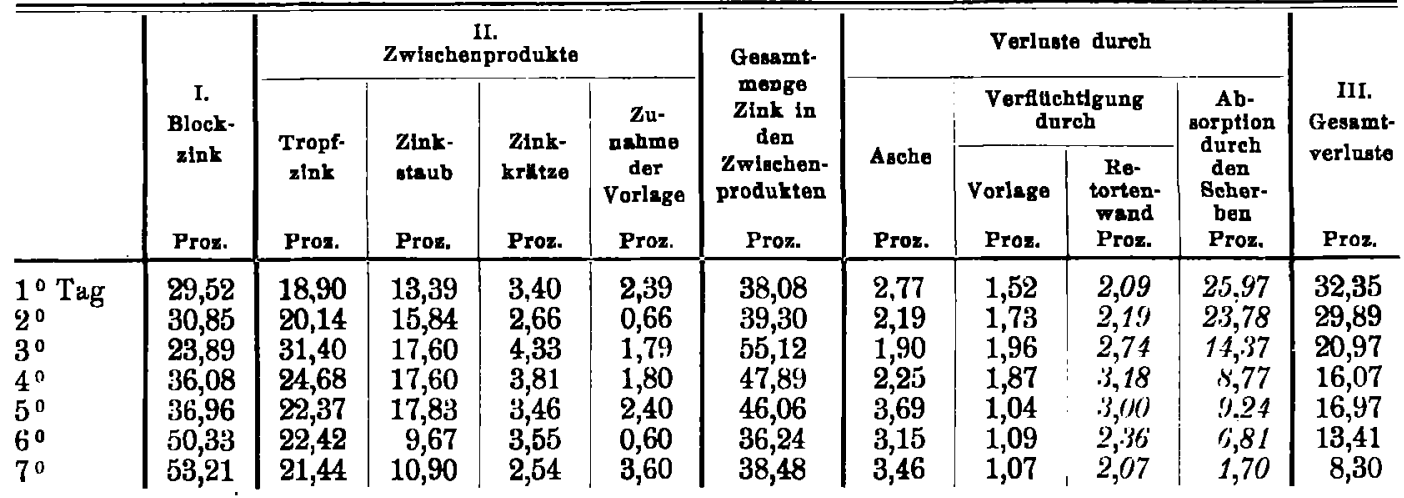

